\title{
Detection of Diethylene Glycol in Glycerin and Propylene Glycol by using high performance thin layer chromatography HPTLC
}

\author{
M.Ph. Huda Ghanem, Prof. Mhd Ammar Alkhayat, Prof. Mhd Amer Almardini \\ Department of pharmaceutical chemistry and quality control, College of Pharmacy, Damascus University, Syria
}

\begin{abstract}
:
We were developed analytical method rapid, sensitive and easy to use to detect Diethylene glycol (DEG) in some of excipients: Glycerin, and propylene glycol by using high-performance thin-layer chromatography HPTLC with plate fluorinated: HPTLC Plate silica gel $60 \mathrm{~F} 254$, and with mobile phase: acetone: toluene: $5 \mathrm{M}$ ammonium hydroxide at rates 85: 5:10, respectively. And Use a scanner with wavelength: $325 \mathrm{~nm}$.

This method allows the detection and assay of toxic Diethylene glycol in excipients: glycerin and propylene glycol in concentrations of not less than $0.1 \%$. This method was verified the validity of it to conduct all the constitutional requirements.
\end{abstract}

Keywords: Diethylene Glycol (DEG), Propylene Glycol (PG), Glycerin, Ethylene Glycol (EG, High Performance Thin Layer Chromatography (HPTLC).

\section{Introduction:}

(DEG) Diethylene Glycol

DEG: ( $\mathrm{HO}-\mathrm{CH} 2-\mathrm{CH} 2-\mathrm{O}-\mathrm{CH} 2-\mathrm{CH} 2-\mathrm{OH})$

Diethylene glycol is Organic solvent has many industrial uses ${ }^{(1,2)}$. DEG is classified as toxic material, it causes when dealing with multiple systemic disorders until the occurrence of acute kidney failure and death ${ }^{(3,4,5)}$. Diethylene glycol has physical and chemical properties close to the properties of glycerin and propylene glycol, which is cheaper than both glycerin and propylene glycol, thus forcing some producers and sellers to cheat them with DEG ${ }^{(6,7,2)}$.

Diethylene glycol is synthesized from the reaction of ethylene oxide with Ethylene glycol, in this case Diethylene glycol can contain Ethylene glycol EG also as toxic impurity ${ }^{(9,8,10)}$.

World Health Organization (WHO) has record since 1937 until 2009 in different countries of the world thousands of cases of poisoning with Diethylene glycol, most of these cases from children, and ended in most cases with death. The reason for this poisoning is dealt with oral pharmaceutical preparations such as syrups, suspensions, Elixirs, and toothpastes which contained Diethylene Glycol as excipient.

As a result of that, most of the organizations and agencies concerned with health, particularly the Food and Drug Administration (FDA) Confirmed the necessary of detection of Diethylene glycol in pharmaceutical preparations and to verify the safety of any drug before marketing ${ }^{(7,11,12,13)}$.

USP, British, European pharmacopeias mentioned in monograph of Glycerin to Gas chromatography method for detection of Diethylene Glycol and Ethylene Glycol in Glycerin as raw material ${ }^{(14,15,16)}$. And there are many reference methods based on high-performance liquid chromatography HPLC ${ }^{(17,18,19,20)}$.

World Health Organization (WHO) and Food and Drug Administration (FDA) had provided a method of thinlayer chromatography TLC (limit method), which were used to detect DEG in Glycerin, Propylene Glycol, and Sorbitol after spray the plate with permanganate solution or iodine.

To be used by institutions and countries which do not have modern equipments which had required to detect DEG as mentioned in pharmacopeias of medicine, this method is an inexpensive, simple and easy to use, but it can not be used in the assay of DEG and determine its quantify in a good accuracy ${ }^{(21,22,23,24)}$.

\section{The study aims to:}

Develop analytical High Performance TLC method quick, sensitive, easy to use, economic, with a validity to detect and assay DEG in excipients: glycerin and propylene glycol, from a mobile phase used on the thin layer which is acetone: toluene: ammonium hydroxide $5 \mathrm{M}$ ratio 85: 5:10, respectively. Proposed by the World Health Organization ${ }^{(21,22,23,24) \text {. }}$

\section{Standards:}

- Standard of Diethylene Glycol DEG: (PROLABO), P (GC): 99\%.

- Standard of Ethylene Glycol EG: (England), P(GC): 99\%.

- Standard of Propylene Glycol PG: (PROLABO), P: 99\%.

- Standard of Glycerin: (England), P (GC): $99-100.5 \%$. 


\author{
Reagents: \\ - Reagents for HPLC: Methanol (Merck). \\ - Acetone (Merck). \\ - Toluene (Merck). \\ - Ammonia Solution 25\% (Merck).
}

\title{
Instruments and tools:
}

High Performance Thin Layer Chromatography: CAMAG HPTLC : CAMAG Automatic TLC Sampler

CAMAG TLC Scanner.

Nitrogen gas.

25 HPTLC aluminum sheets (Merck), 20 x $20 \mathrm{~cm}$ silica gel $60 \mathrm{~F}_{254}$.

CAMAG, HPTLC Immersion Device.

Sartorius Analytic Balance (0.0001 mg).

\section{Preparation of solutions:}

Stock standard solution of DEG in Methanol with concentration: $2 \mathrm{mg} / \mathrm{ml}$.

Standard solution of DEG in Methanol with concentration: $0.5 \mathrm{mg} / \mathrm{ml}$.

Sample solution of raw material in Methanol with concentration: $500 \mathrm{mg} / \mathrm{ml}$.

Preparation of Validation solution ${ }^{(14,19,25)}$.

\section{Chromatographic system:}

Mobile phase: mixture of Acetone: Toluene: Ammonium Hydroxide 5 M (85:5:10) respectively HPTLC Plate silica size: $10 \times 10 \mathrm{~cm}$. and drying plate after chromatography with air.

Spray gas: nitrogen.

Injection volume: $2 \mu 1$.

Wavelength: $325 \mathrm{~nm}$.

We calculated the percentage of DEG in the samples accordance with the monograph of glycerine in USP, and British Pharmacopoeia ${ }^{(14,15)}$, and it shouldn't be more than $0.1 \%$ from DEG of the weight of samples ${ }^{(14,15)}$.

\section{Results}

When we inject each of Diethylene glycol, Ethylene glycol, glycerine, propylene glycol, and Polyethylene glycol 400 on TLC plate in the conditions of the method which we used, and identify the values of Retention Factor RF for each of them (Table 1). The Retention Factor RF of Ethylene glycol is close to The Retention Factor RF of Diethylene glycol, and we cannot distinguish between them if they were together in same sample, so we excluded Ethylene Glycol from this study as shown in Figure 1. The Retention Factor RF of Poly Ethylene glycol 400 is so closed to The Retention Factor RF of Diethylene glycol therefore we cannot use this method to detect DEG in the sample of PEG 400 as in (Figure 2).

Figure 3 shows chromatogram of standard solution DEG ( $2 \mu \mathrm{l}$ of $0.5 \mathrm{mg} / \mathrm{ml} \mathrm{DEG})$. And Table 2 shows areas of peaks resulting from injecting the standard solution for five spots in a row and the value of the relative standard deviation RSD.

Figures 4.5 show chromatograms of Glycerin sample and Propylene Glycol sample respectively $(2 \mu \mathrm{lof} 500 \mathrm{mg} / \mathrm{ml})$. And Figures 6.7 show chromatograms of Glycerin sample and Propylene Glycol sample respectively that containing standard DEG with concentration $(0.5 \mathrm{mg} / \mathrm{ml})$.

\section{Results of Analytical Methods Validation}

\section{Accuracy:}

The average percentage of recovery is: $106.36, \% 103.89$ for samples solutions of glycerine and PG respectively, with concentrations: $(50 \%, 100 \%, 120 \%$ of DEG standard).

\section{Precision:}

The average percentage of recovery is: $103.87 \%, \% 100.59$ for samples solutions of glycerine and PG respectively, three samples with each concentration: (50\%, 100\%, $120 \%$ of DEG standard), and the value of RSD to these recoveries is $10.81 \%$, 10.81 for samples of glycerine and PG respectively. 


\section{Selectivity:}

When we inject placebo sample of glycerine or PG didn't contain DEG, there were no response occur in retention time of DEG. And the average of percentage of recovery is: $103.19 \%, \% 97.74$ for three samples solutions of glycerine or PG with concentrations: $100 \%$ of DEG standard respectively.

\section{Linearity \& Range:}

We recorded the responses of each concentration of DEG Standard (50\%, 75\%, 100\%, 125\%, 150\%) (Table: 3), the Linear Regression Equation corresponding to these responses (Figure: 8), and the value of the Correlation Factor is: 0.9971.

\section{Detection limit:}

Detection limit is equal to $0.033 \mathrm{mg} / \mathrm{ml}$, equivalent to $6.6 \%$ of the standard concentration.

\section{Quantification Limit:}

Quantification limit is equal to $0.109 \mathrm{mg} / \mathrm{ml}$, equivalent to $21.8 \%$ of the standard concentration

\section{Robustness:}

The average percentage of recovery for DEG in the samples of Glycerine is: $100.6 \%, 100.3 \%, 101.53 \%$, respectively, with the change of the distance which the mobile phase was reached, in the order $(80,90,100 \mathrm{~mm})$.

As well as for the average percentage of recovery for DEG in the samples of propylene glycol is: $102.7 \%, 101.19 \%, 98.71 \%$, respectively, with the same changing in distance as in case of Glycerine.

The Relative retention times of DEG for Glycerine samples is: 1.0, 0.91, 0.98 respectively with the previous changing, and The Relative retention times of DEG for Propylene Glycol samples is: 0.98, 0.88, 0.92 respectively also with the previous changing.

\section{Discussion and Conclusions:}

The results of verification tests had shown that the studied method of HPTLC meet the requirements of validation in the Pharmacopoeia ${ }^{(17.18)}$, while the percentages of recovery in tests, accuracy and specificity for Glycerine samples are: $106.4 \%$, $103.19 \%$, respectively, and for Propylene Glycol samples are: 103.8\%, 97.74\% respectively.

Also the relative standard deviation RSD of values of recovery in tests of repeatability for Glycerine and Propylene Glycol are: $10.8 \%, 5.1 \%$ respectively.

The results also had shown that this method is linear, and the correlation coefficient is close to one: 0.9971 (Figure 8, Table 8).

And the value of detection limit is: $0.033 \mathrm{mg} / \mathrm{ml}$, and the value of Quantification limit is $0.109 \mathrm{mg} / \mathrm{ml}$.

We can by using the applied method of HPTLC, detect and assay DEG as an impurity in glycerin and propylene glycol. And this method is rapid, sensitive and inexpensive and does not require the completion of any analysis of more than small TLC plate dimensions of $10 \times 10 \mathrm{~cm}$. And the time required completing of any analysis takes only approximately one hour, .including preparation of solutions and injected and the deportation of the mobile phase on the plate 


\section{Appendix of tables and chromatograms:}

Table (1): Retention factor of DEG \& EG \& PG \& PEG 400 \& Glycerin:

\begin{tabular}{|c|c|c|c|c|c|}
\hline Name & DEG & EG & PG & Glycerin & PEG 400 \\
\hline Retention Factor $\mathrm{R}_{\mathrm{F}}$ & 0.40 & 0.44 & 0.69 & 0.04 & 0.43 \\
\hline
\end{tabular}

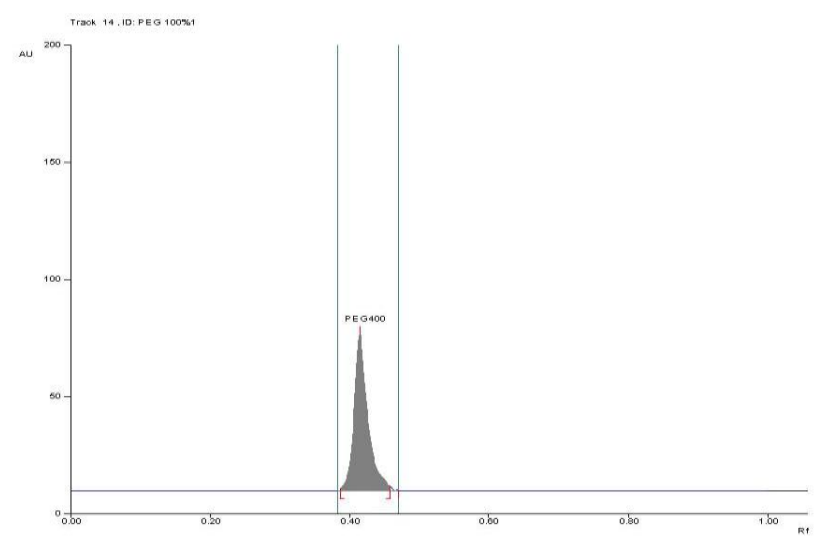

Figure (2): Chromatogram of sample solution of PEG400

Table (2): relative standard deviation of area of

\begin{tabular}{|c|c|c|c|c|}
\hline $\begin{array}{c}\text { Standard. } \\
\text { NO }\end{array}$ & Area & $\mathrm{R}_{\mathrm{f}}$ & & \\
\hline Std - 1 & 555 & 0.40 & & \\
\hline Std - 2 & 548 & 0.39 & & \\
\hline Std - 3 & 544 & 0.39 & Average & 560.8 \\
\hline Std - 4 & 571 & 0.41 & SD & 17.5 \\
\hline Std - 5 & 586 & 0.39 & RSD & 3.11 \\
\hline
\end{tabular}

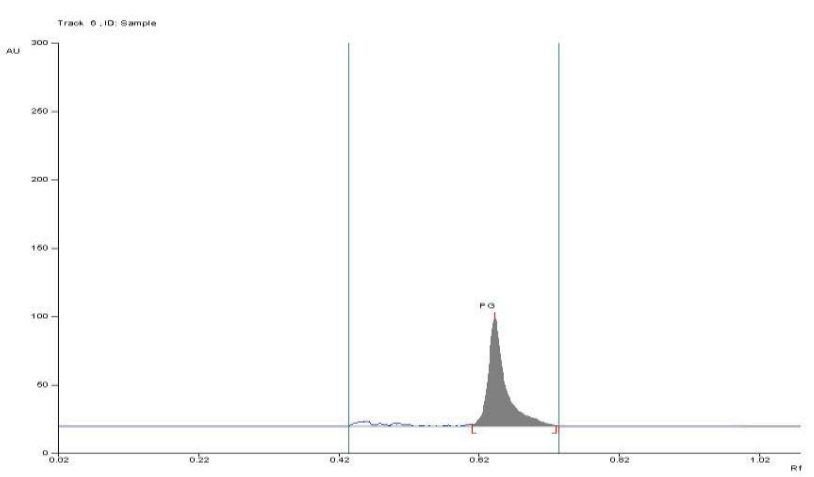

Figure (5): Chromatogram of sample solution of PG

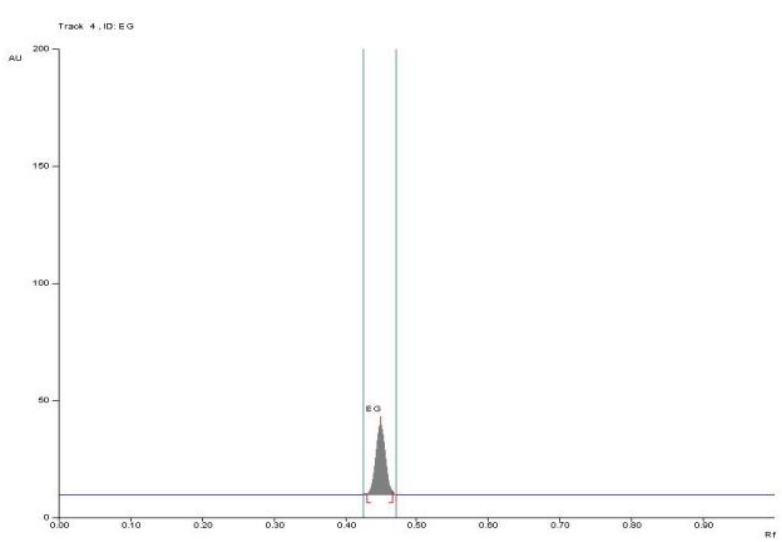

Figure (1): Chromatogram of solution of EG

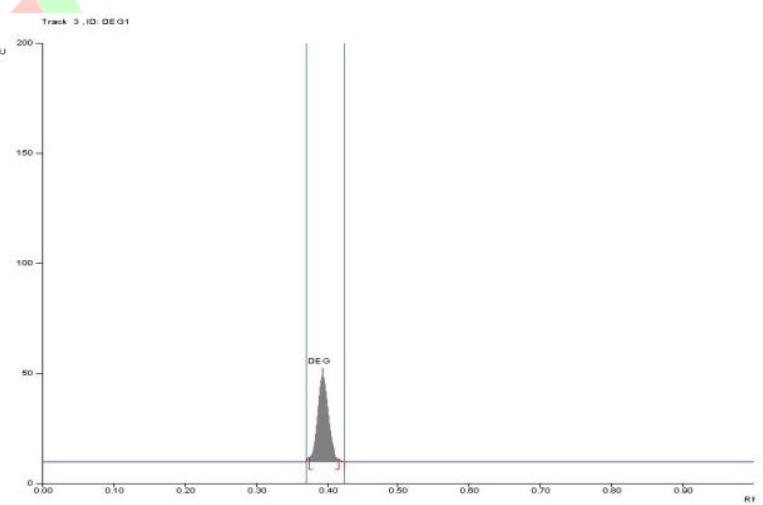

Figure (3): Chromatogram of standard solution DEG

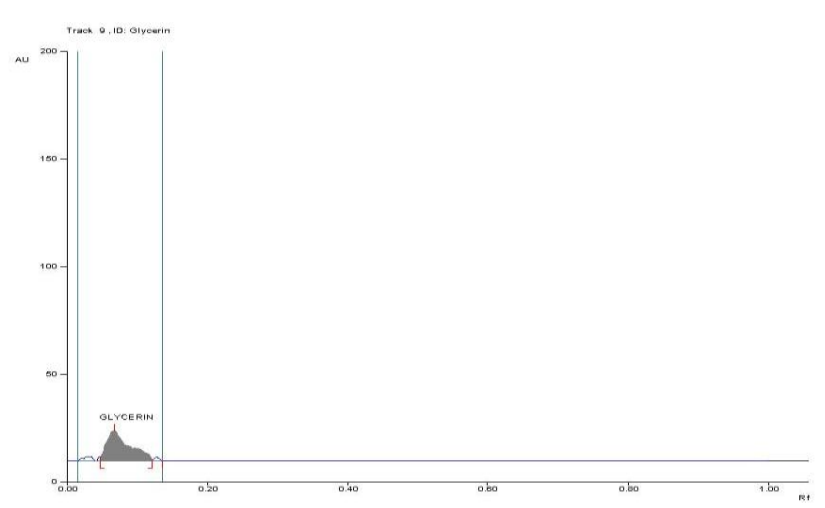

Figure (4): Chromatogram of sample solution of Glycerin 


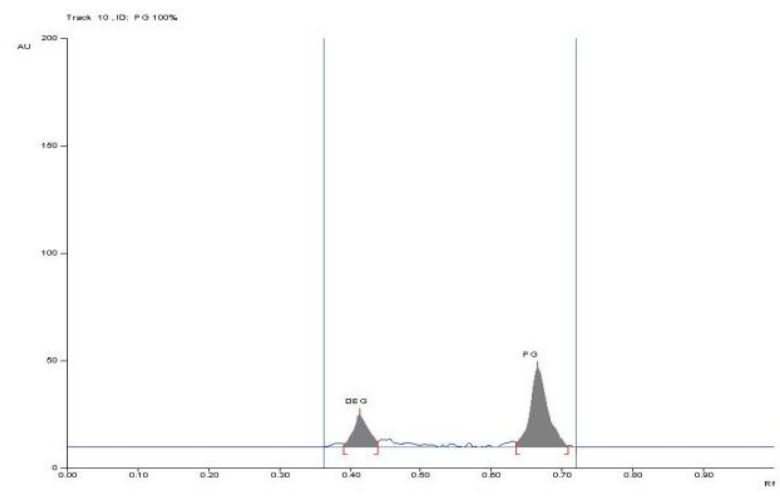

Figure (7): Chromatogram of sample solution of PG with $100 \%$ St Concentration

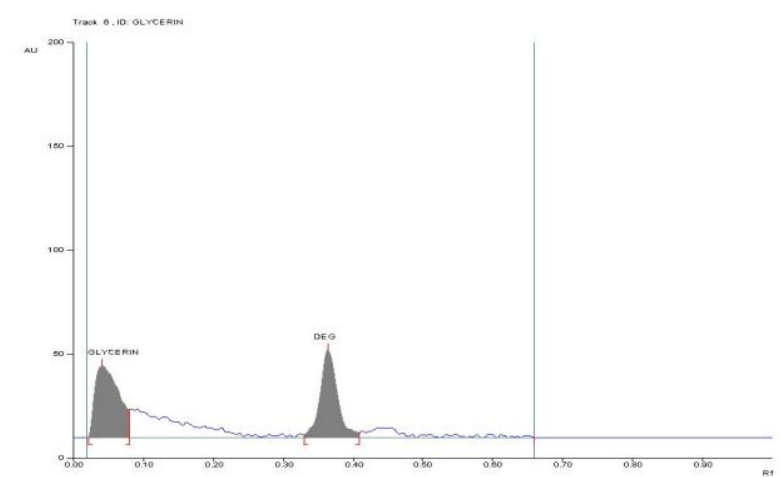

Figure (6): Chromatogram of sample solution of Glycerin with $100 \%$ St Concentration

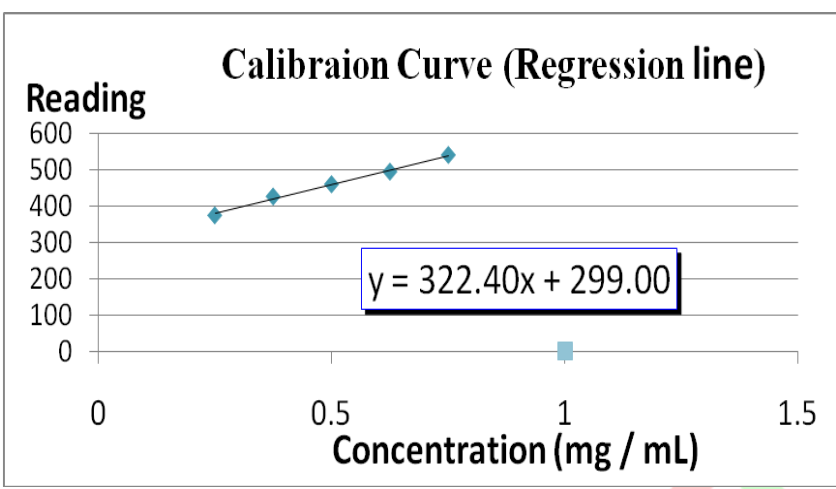

Table (3): result of Linearity of method

Figure (8): Chromatogram of Linear Regression Equation

\section{References}

1- Jeanna M. Marraffa \& Michael G. Holland \& Christine M. Stork \& Christopher D. Hoy\& Michael J. Hodgman ,'The Journal of Emergency Medicine", Diethylene Glycol: Widely Used Solvent Presents Serious Poisoning Potential, Vol. 06, No. 025, 17 February 2007.

2- $\quad$ James E.F.Reynolds \& Kathleem Parfitt \& Anne V.Parsons \& Sean C. Sweetman, “ Martindale, The Complete Drugs Reference “. Thirty - Fifth Edition: 2007 ; (1754 - 1756, 2100, 2152, 2118, 2088).

3- $\quad$ Ijeoma F. Uchegbu \& Andreas G. Schatzlein, “ Polymer in Drug Delivery “. 2006 ; (172 - 177).

4- $\quad$ Kevin McClay \& Charles E. Schaefer \& Simon Vainberg \& Robert J. Steffan, “ American Society for Microbiology “, Biodegradation of Bis(2-chloroethyl) Ether by Xanthobacter sp , Vol. 73, No.21 ;Nov. 2007, p. $6870-6875$.

5- Jeffrey A. Kraut \& Ira Kurtz, “ Journal American Society of Nephrology, Toxic Alcohol Ingestions: Clinical Features, Diagnosis, and Management” 3: 208-225, 2008.

6- $\quad$ M. Lopez-Sanchez \& A. Dominguez-Vidal \& M.J. Ayora-Canada \& A. Molina-Diaz," Analytical Chemica Acta ", Assessment of dentifrice adulteration with diethylene glycol by means of ATR-FTIR spectroscopy and chemometrics, Volume 620, Issues 1-2, 14 July 2008, Pages 113-119.

7- David A. Warrell \& Edward J. Benz,Jr \& Timothy M. Cox \& John D. Firth, "Oxford Textbook of Medicine “. Fourth Edition: 2003 ; part 1,913, 894-898), (part 3,470).

8- $\quad$ Parveen Kumar \& Michael Clark, "Kumar \& Clark, Clinical Medicine “. Sixth Edition: 2007 ; (322, 1012 - 1013, 1102).

9- $\quad$ Lee Goldman \& Dennis Ausiello, “Cecil, Medicine “. $23^{\text {th }}$ Edition: $2008 ;(774-779,787,853,1761$, 2935).

10- Dr. Wayne A Temple, "International Programme on Chemical Safety “, DEG - draft poisons information monograph for peer review ; October, 2007. 
11- E Danielle Rentz \& Lauren Lewis \& Oscar J Mujica \& Dana B Barr \& Joshua G Schier \& Gayanga Weerasekera \& Peter Kuklenyik \& Michael McGeehin \& John Osterloh, "Bulletin of The World Health Organization (BLT), Outbreak of acute renal failure in Panama in 2006: a case-control study " Volume 86, Number 10, October 2008, 737-816.

12- Esther Perez \& Jacobo Limeres \& Inmaculada Tomas, “ Med Oral Patol Oral Cir Bucal " Toothpaste with DEG, 2008 Apr 1;13(4): E222-3.

13- Marketwire ," Health Canada, Update on foreign toothpaste containing DEG " 2008-81; May 28,2008 .

14- United States Pharmacopoeia (USP 30 -2009), (911 - 912, 1665, 2748 - 2751, 2386 - 2392, 3000 3005, 2492, 3053 - 3054, 3012 - 3015, 3055 - 3057).

15- $\quad$ British Pharmacopoeia (BP 2009), (759 - 760, 1392, 974 - 975, 762, 527 - 529, 645).

16- European Pharmacopoeia (EP 2007).

17- $\quad$ Leo M.L.NO uet \& Hoyeschool Gent, “Food Analysis by HPLC “. Second Edition: 2000 ; (303 319 ).

18- Tao Zhou \& Haiying Zhang \& Gengli Duan, "Journal of Separation Science”, Simultaneous determination of DEG and propylene glycol in pharmaceutical products by HPLC after precolumn derivatization with p-toluenesulfonyl isocyanate, 14 July 2007, Volume 30 Issue 16, Pages 2620 2627.

19- Anthony C Moffat \& M David Osselton \& Brian Widdop, "Clarke's Analysis of Drugs and Poisons “. Third Edition : 2004 ; (14, 242, 1001, 273 - 278, 161 - 171, 98 - 108, 425 - 445, 500 - 504, 161164).

20- $\quad$ Luis A. Ferrari \& Leda Giannuzzi, "Forensic Science International “, Clinical parameters, postmortem analysis and estimation of lethal dose in victims of a massive intoxication with diethylene glycol : 4 Oct $2005 ; 153$ (1): 45-51.

21- Katherine L. O’Brien \& Joel D. Selanikio \& Charleine Hecdivert, "JAMA, Journal American Medical Association, Epidemic of pediatric deaths from acute renal failure caused by DEG poisoning “, April 15, 1998- Vol 279,NO. 15; (1175-1180).

22- Jagvir Singh \& Shashi khare \& A.K. Dutta \& N.K. Dubey \& A.K. Harit, "Bulletin of The World Health Organization, DEG poisoning in Gurgaon India 1998 “, $2001 ; 79: 88$ - 95.

23- Allen S. Kenyon \& Shi Xiaoye \& Wang Yan \& Ng Wai Har, "Journal of AOAC International, Simple, At-Site Detection of DEG/EG contamination of Glycerin and Glycerin-Based raw materials by TLC “Vol. 81. NO. 1. $1998 ;(44-50)$.

24- Rockville, Center for Drug Evaluation and Research, "FDA, U.S. Food and Drug Administration ", Guidance for Industry, Testing of Glycerin for DEG, May 1, 2007.

25- Mhd Amer Almardini, “quality control”, Damascus university. 2007-2008 (615-632). 